\title{
Characterization and comparison of Sugeno and Choquet integrals
}

\author{
Luis M. de Campos and M. Jorge \\ Bolaños \\ Departamento de Ciencias de la Computación e Inteligencia \\ Artificial, Facultad de Ciencias, Universidad de Granada, \\ Spain \\ Received April 1991 \\ Revised December 1991
}

Abstract: Starting from the concept of 'equiordered functions' we characterize two types of fuzzy integrals that can be defined on any kind of fuzzy measure: Sugeno and Choquet integrals. The characterization theorems that we obtain allow us to carry out a comparative study of these two fuzzy integrals, pointing out their formal similarities and their conceptual differences.

Keywords: Fuzzy measure; fuzzy integral; Choquet integral; Sugeno integral.

\section{Introduction}

If a fuzzy measure is available on a referential, it is interesting to have tools able to summarize all the pieces of information provided by a function in a single value; this value would be a sort of average of the function, in terms of the underlying fuzzy measure. Such tools are the fuzzy integrals. The purpose of this paper is to characterize and compare two types of fuzzy integrals: Sugeno integral and Choquet integral (also called monotone expectation). These two functionals, unlike others $[2,15,24,25,27]$, can be defined on any kind of fuzzy measure.

The paper is arranged in three sections: The definitions of Sugeno and Choquet integrals, along with their most outstanding and wellknown properties are described in Section 2. We also obtain other properties by using the concept

Correspondence to: Dr. Luis M. de Campos Ibáñez, Departamento de Ciencias de la Computación e Inteligencia Artificial, Facultad de Ciencias, Universidad de Granada, 18071 Granada, Spain. of 'equiordered functions', which is considered basic for the further developments.

Characterizations of the considered integrals are proved in Section 3. These characterizations allow us to carry out an interesting comparative study of Choquet and Sugeno integrals, in order to point out their formal similarities and their conceptual differences.

\section{Definitions and basic properties}

Let $X=\left\{x_{1}, x_{2}, \ldots, x_{n}\right\}$ be a finite referential. The following definition reflects Sugeno's concept of fuzzy measure for the finite case [21]:

Definition 2.1. A fuzzy measure on $X$ is a set function

$g: \mathscr{P}(X) \rightarrow[0,1]$,

where $\mathscr{P}(X)$ is the power set of $X$, satisfying

(i) $g(\emptyset)=0 ; g(X)=1$.

(ii) $\forall A, B \subseteq X$, if $A \subseteq B$ then $g(A) \leqslant g(B)$.

Therefore a fuzzy measure is a monotone and normalized set function. It could be considered as an extension of the concept of probability measure, replacing the additivity by the weaker condition of monotonicity.

Remark. Here we will only consider the case of a finite referential $X$. The main reason to restrict our study to this case is that continuous variables and sets involve a number of purely mathematical difficulties and restrictions (e.g. measurability and continuity) which not only result in rather high technical demands but, more importantly, obscure the important issues. There are also some other different reasons justifying the use of discrete universes and variables; for example, because of the unavoidable finite errors in observations, data are always discrete, regardless 
of philosophical beliefs or the state of technology. When these kinds of empirical considerations are not dominating and the use of continuous variables or sets is desirable, an appropriate finite resolution level can always be chosen which approximate the continuous variable or universe as closely as one wishes (see Klir [14] for a detailed discussion of the dichotomy of discrete versus continuous sets). Anyway, the extension to the case of infinite universes of the results obtained here will be considered in further works.

The most well-known and used fuzzy integral was defined by Sugeno [21], as follows:

Definition 2.2. Let $g$ be a fuzzy measure on $X$ and $h: X \rightarrow[0,1]$ a function. The Sugeno integral of $h$ with respect to $g$ is

$f h \circ g=S_{g}(h)=\sup _{0 \leqslant \alpha \leqslant 1}\left(\alpha \wedge g\left(H_{\alpha}\right)\right)$,

where $H_{\alpha}=\{x \in X \mid h(x) \geqslant \alpha\}$ and the minimum and maximum operators are denoted by the symbols $\wedge$ and $\vee$, respectively.

The Sugeno integral has been deeply studied by several authors from different points of view. Most noteworthy among such studies are the works of Batle and Trillas [1], Bolaños [3], Ralescu and Adams [18], who obtained several characterizations of this fuzzy integral.

Its most important properties are;

1. If $a \in[0,1]$, then $S_{g}(a)=a$.

2. If $h(x) \leqslant h^{\prime}(x) \forall x \in X\left(h \leqslant h^{\prime}\right.$ in short), then $S_{g}(h) \leqslant S_{g}\left(h^{\prime}\right)$.

3. If $g(A) \leqslant g^{\prime}(A) \quad \forall A \subseteq X$, then $S_{g}(h) \leqslant$ $S_{g^{\prime}}(h) \forall h: X \rightarrow[0,1]$.

4. $S_{g}\left(I_{A}\right)=g(A)$, where $I_{A}$ is the characteristic function of $A \subseteq X$.

5. If $a \in[0,1]$, then, $\forall h: X \rightarrow[0,1]$,

$S_{g}(a \vee h)=a \vee S_{g}(h)$,

$S_{g}(a \wedge h)=a \wedge S_{g}(h)$.

Proofs of these properties can be found in [21]

Another fuzzy integral is the Choquet integral [8] (called monotone expectation by Bolaños et al. [4], to emphasize its parallelism with the mathematical expectation, but losing additivity and preserving monotonicity). Although the Choquet integral was introduced in 1953 and was used in Statistics (see $[10,11]$ ), only recently it has been considered by the fuzzy community (see $[4,5,6,7,17,23]$ ).

Definition 2.3. Let $g$ be a fuzzy measure on $X$ and $h: X \rightarrow \mathbb{R}_{0}^{+}$a non-negative function. The Choquet integral of $h$ with respect to $g$ is

$E_{g}(h)=\int_{0}^{+\infty} g\left(H_{\alpha}\right) \mathrm{d} \alpha$,

where $H_{\alpha}=\{x \in X \mid h(x) \geqslant \alpha\}$.

The Choquet integral always exists (for the case of finite universes), and it is obviously a generalization of the mathematical expectation: which is what it becomes when the fuzzy measure $g$ is a probability measure.

The most important properties of this functional are:

1. If $a \in \mathbb{R}_{0}^{+}$, then $E_{g}(a)=a$.

2. If $h(x) \leqslant h^{\prime}(x) \quad \forall x \in X$, then $E_{g}(h) \leqslant$ $E_{g}\left(h^{\prime}\right)$.

3. If $g(A) \leqslant g^{\prime}(A) \quad \forall A \subseteq X$, then $E_{g}(h) \leqslant$ $E_{g^{\prime}}(h) \forall h: X \rightarrow \mathbb{R}_{0}^{+}$.

4. $E_{g}\left(I_{A}\right)=g(A)$.

5. If $b, c \in \mathbb{R}_{0}^{+}$, then $E_{g}(c+b h)=c+b E_{g}(h)$ $\forall h: X \rightarrow \mathbb{R}_{0}^{+}$.

6. The Choquet integral is not an additive functional in general; it is additive only for probability measures.

Proofs of these properties can be found for example in $[4,5]$. Moreover, the upper and lower integrals, defined by Dempster [9], are particular cases of the Choquet integral for fuzzy measures that are plausibility and belief functions, respectively (see $[4,9,19]$ ).

Hereafter we shall denote the value of a function $h$ at point $x_{i} \in X$ by $h_{i}$. If the values of the function $h$ satisfy $h_{1} \leqslant h_{2} \leqslant \cdots \leqslant h_{n}$, it becomes easy to verify (taking into account the form of the sets $H_{\alpha}$ ) that Sugeno integral and Choquet integral can be written as follows (see [7]):

$$
\begin{aligned}
& S_{g}(h)=\bigvee_{i=1}^{n}\left(h_{i} \wedge g\left(A_{i}\right)\right), \\
& E_{g}(h)=\sum_{i=1}^{n} h_{i}\left(g\left(A_{i}\right)-g\left(A_{i+1}\right)\right),
\end{aligned}
$$


where

$A_{i}=\left\{x_{i}, x_{i+1}, \ldots, x_{n}\right\}, \quad i=1, \ldots, n$,

and $A_{n+1}=\emptyset$.

Equation (2) leads us to consider the following fact: two functions having the same ordering of their values generate the same associated sets $A_{i}$ that we need for the calculation of the integral and whose form depends only on this order; hence the Choquet integral of their sum is the sum of both Choquet integrals, because the sum function ranks its values in the same way. Therefore the Choquet integral is an additive functional for that kind of functions (see $[6,7,17,23])$.

To formulate this idea more precisely, we define a binary relation between functions, which we call 'equiordering', as follows:

Definition 2.4. Given two functions $h$ and $h^{\prime}, h$ is said to be 'equiordered' with $h^{\prime}$ ', and it is denoted by $h \approx h^{\prime}$, if and only if $h$ is a constant function or, for each pair $x_{i}, x_{j} \in X$ such that $h_{i}<h_{j}$, it follows necessarily that $h_{i}^{\prime} \leqslant h_{j}^{\prime}$.

This definition attempts to reflect the idea that the values of both functions $h$ and $h^{\prime}$ are ordered in the same way, on the understanding that any order can be considered when two or more values of the function are equal.

It is easy to verify that the equiordering relation, while having the reflective and symmetric properties, lacks the transitive one; therefore, $\simeq$ is not an equivalence relation. This happens because the definition is not based on strict inequalities, and allows a constant function to be considered equiordered with any other function.

It is also very easy to prove that the relation $\simeq$ is compatible with any monotone binary operation $*$ on positive real numbers, that is to say, if $h \simeq h^{\prime \prime}$ and $h^{\prime} \simeq h^{\prime \prime}$ then $\left(h * h^{\prime}\right) \simeq h^{\prime \prime}$.

Starting from the equiordering relation, the additivity of the Choquet integral for equiordered functions can be formalized as shown in the following proposition:

Proposition 2.1. Let $h, h^{\prime}: X \rightarrow \mathbb{R}_{0}^{+}$be two non-negative functions, and let $g$ be a fuzzy measure on $X$. If $h$ and $h^{\prime}$ are equiordered functions, then

$$
E_{g}\left(h+h^{\prime}\right)=E_{g}(h)+E_{g}\left(h^{\prime}\right) .
$$

Proof. For convenience, let us suppose that $h_{1} \leqslant h_{2} \leqslant \cdots \leqslant h_{n}$. As $h \approx h^{\prime}$ then $h_{1}^{\prime} \leqslant h_{2}^{\prime} \leqslant$ $\cdots \leqslant h_{n}^{\prime}$ too, and therefore

$h_{1}+h_{1}^{\prime} \leqslant h_{2}+h_{2}^{\prime} \leqslant \cdots \leqslant h_{n}+h_{n}^{\prime}$.

If the ordering of the values of $h$ is different, a suitable modification of the notation of the elements of $X$ would suffice to obtain the same result.

In these conditions, with $A_{i}=$ $\left\{x_{i}, x_{i+1}, \ldots, x_{n}\right\}$ and taking into account (2), we obtain

$$
\begin{aligned}
E_{g}\left(h+h^{\prime}\right)= & \sum_{i=1}^{n}\left(h_{i}+h_{i}^{\prime}\right)\left(g\left(A_{i}\right)-g\left(A_{i+1}\right)\right) \\
= & \sum_{i=1}^{n} h_{i}\left(g\left(A_{i}\right)-g\left(A_{i+1}\right)\right) \\
& +\sum_{i=1}^{n} h_{i}^{\prime}\left(g\left(A_{i}\right)-g\left(A_{i+1}\right)\right) \\
= & E_{g}(h)+E_{g}\left(h^{\prime}\right) .
\end{aligned}
$$

Therefore, although the Choquet integral is not an additive functional, it does have an additivity property for equiordered functions, which we call 'ordered additivity'.

The Sugeno integral also verifies an analogous property in relation to the equiordering relation:

Proposition 2.2. Let $h, h^{\prime}: X \rightarrow[0,1]$ be two functions and let $g$ be a fuzzy measure on $X$. If $h$ and $h^{\prime}$ are equiordered functions, then

$S_{g}\left(h \vee h^{\prime}\right)=S_{g}(h) \vee S_{g}\left(h^{\prime}\right)$.

Proof. As $h \simeq h^{\prime}$, if we again suppose that $h_{1} \leqslant h_{2} \leqslant \cdots \leqslant h_{n}$, then $h_{1}^{\prime} \leqslant h_{2}^{\prime} \leqslant \cdots \leqslant h_{n}^{\prime}$ and

$h_{1} \vee h_{1}^{\prime} \leqslant h_{2} \vee h_{2}^{\prime} \leqslant \cdots \leqslant h_{n} \vee h_{n}^{\prime}$.

Using the distributivity of the minimum operator with respect to the maximum operator, 
and using (1), we obtain

$$
\begin{aligned}
S_{g}\left(h \vee h^{\prime}\right) & =\bigvee_{i=1}^{n}\left(\left(h_{i} \vee h_{i}^{\prime}\right) \wedge g\left(A_{i}\right)\right) \\
& =\bigvee_{i=1}^{n}\left(\left(h_{i} \wedge g\left(A_{i}\right)\right) \vee\left(h_{i}^{\prime} \wedge g\left(A_{i}\right)\right)\right) \\
& =\bigvee_{i=1}^{n}\left(h_{i} \wedge g\left(A_{i}\right)\right) \vee \bigvee_{i=1}^{n}\left(h_{i}^{\prime} \wedge g\left(A_{i}\right)\right) \\
& =S_{g}(h) \vee S_{g}\left(h^{\prime}\right) .
\end{aligned}
$$

Hence the Sugeno integral verifies a weaker property than F-additivity, which we call ordered F-additivity, by analogy with the ordered additivity property for the Choquet integral.

\section{Characterizations of Choquet and Sugeno integrals}

Even though the properties that characterize the mathematical expectation are well-known, i.e. additivity and normalization, it is interesting to study what properties would be sufficient to characterize the Choquet integral functional, when the additivity is replaced by the ordered additivity. The following theorem gives and answer to this question:

Theorem 3.1. A functional $E$ defined on non-negative functions satisfies the conditions:

(a) Ordered additivity: if $h \simeq h^{\prime}$, then $E(h+$ $\left.h^{\prime}\right)=E(h)+E\left(h^{\prime}\right)$,

(b) Monotonicity: if $h \leqslant h^{\prime}$, then $E(h) \leqslant$ $E\left(h^{\prime}\right)$,

(c) Normalization: $E\left(I_{X}\right)=1$,

(d) Homogeneity: $\forall a>0 E(a h)=a E(h)$,

if and only if there exists only one fuzzy measure $g$ such that $E$ is the Choquet integral with respect to $g$.

Proof. Necessity: Let us define the set function $g(A)=E\left(I_{A}\right) \forall A \subseteq X$. First, we will prove that $g$ is a fuzzy measure:

(i) $g(X)=E\left(I_{X}\right)=1$ by condition (c).

$g(\emptyset)=E\left(I_{\emptyset}\right)=E\left(0_{X}\right)=0$

because of the following reasoning:

As $0_{X}$ is a constant function, $0_{X} \approx h$ for every function $h$. By using condition (a), we obtain

$E(h)=E\left(h+0_{X}\right)=E(h)+E\left(0_{X}\right)$,

whence we conclude that $E\left(0_{X}\right)=0$.

(ii) If $A \subseteq B$, then $I_{A}(x) \leqslant I_{B}(x) \forall x \in X$, and, from condition (b),

$g(A)=E\left(I_{A}\right) \leqslant E\left(I_{B}\right)=g(B)$.

Therefore $g$ is a fuzzy measure.

We shall now prove that $E(h)=E_{g}(h)$ for every non-negative function $h$ :

Consider a non-negative function $h$ and let us suppose that it verifies $h_{1} \leqslant h_{2} \leqslant \cdots \leqslant h_{n}$, which is not a restriction. Then $h$ can be expressed as

$h(x)=\sum_{i=1}^{n} f_{i}(x)$,

where

$f_{i}(x)=\left(h_{i}-h_{i-1}\right) I_{A_{i}}(x), \quad i=2, \ldots, n$,

$f_{1}(x)=h_{1} I_{A_{1}}(x)$,

and

$A_{i}=\left\{x_{i}, x_{i+1}, \ldots, x_{n}\right\}, \quad i=1, \ldots, n$.

It is easy to confirm that the functions $f_{i}$ are equiordered between each other, that is, $f_{i} \simeq f_{j}$ $\forall i, j=1, \ldots, n$.

By repeatedly applying the compatibility of the equiordering relation with respect to the sum (which is a monotone operation), and using (a) and (d), we obtain

$$
\begin{aligned}
E(h) & =E\left(\sum_{i=1}^{n} f_{i}\right)=\sum_{i=1}^{n} E\left(f_{i}\right) \\
& =E\left(h_{1} I_{A_{1}}\right)+\sum_{i=2}^{n} E\left(\left(h_{i}-h_{i-1}\right) I_{A_{i}}\right) \\
& =h_{1} E\left(I_{A_{1}}\right)+\sum_{i=2}^{n}\left(h_{i}-h_{i-1}\right) E\left(I_{A_{i}}\right) \\
& =h_{1}+\sum_{i=2}^{n}\left(h_{i}-h_{i-1}\right) g\left(A_{i}\right) \\
& =h_{n} g\left(A_{n}\right)+\sum_{i=1}^{n-1} h_{i}\left(g\left(A_{i}\right)-g\left(A_{i+1}\right)\right) \\
& =E_{g}(h) .
\end{aligned}
$$

Finally, uniqueness of $g$ is evident.

Sufficiency: It is obvious, since conditions (b), (c) and (d) are known properties of $E_{g}(\cdot)$, and condition (a) was proved in Proposition 2.1. 
Therefore, the Choquet integral is characterized as an equiordered, monotone, normalized and homogeneous functional.

As the basic properties of the Sugeno integral are equal or similar to those of the Choquet integral, replacing sum and product by maximum and minimum, it is reasonable to look for a characterization of the Sugeno integral similar to that established for the Choquet integral in Theorem 3.1:

Theorem 3.2. A non-negative functional $E$, defined for functions taking their values in the interval $[0,1]$, satisfies the conditions:

(a') Ordered F-additivity: If $h \simeq h^{\prime}$ then $E\left(h \vee h^{\prime}\right)=E(h) \vee E\left(h^{\prime}\right)$,

(b) Monotonicity: If $h \leqslant h^{\prime}$ then $E(h) \leqslant$ $E\left(h^{\prime}\right)$,

(c) Normalization: $E\left(I_{X}\right)=1$,

(d') $\forall a \in(0,1] E(a \wedge h)=a \wedge E(h)$,

if and only if there exists only one fuzzy measure $g$ such that $E$ is the Sugeno integral with respect to $g$.

Proof. Sufficiency: It follows immediately from the properties of the Sugeno integral and Proposition 2.2.

Necessity: Let us define again the set function $g(A)=E\left(I_{A}\right) \forall A \subseteq X$ as in Theorem 3.1. We will prove that $g$ is a fuzzy measure.

The monotonicity of $g$ is directly deduced from condition (b), and $g(X)=1$ follows from condition (c). It remains to be proved that $g(\emptyset)=0$ :

$$
\forall a \in(0,1], \quad \begin{aligned}
E(a) & =E\left(a \wedge I_{X}\right)=a \wedge E\left(I_{X}\right) \\
& =a \wedge 1=a,
\end{aligned}
$$

from conditions $(c)$ and $\left(d^{\prime}\right)$; then, by using $\left(a^{\prime}\right)$, we obtain

$$
\forall a \in(0,1], \quad \begin{aligned}
a & =E(a)=E\left(a \vee 0_{X}\right) \\
& =E(a) \vee E\left(0_{X}\right)=a \vee E\left(0_{X}\right) .
\end{aligned}
$$

Hence $E\left(0_{X}\right) \leqslant a \forall a \in(0,1]$, and we can state that $g(\emptyset)=E\left(0_{X}\right)=0$ because $E$ is a nonnegative functional.

Now, we will prove that $E(\cdot)$ coincides with $s_{g}(\cdot)$.

Let $h: X \rightarrow[0,1]$ and let us suppose (without lack of generality) that $h_{1} \leqslant h_{2} \leqslant \cdots \leqslant h_{n}$. Then $h$ can be written as

$h(x)=\bigvee_{i=1}^{n} t_{i}(x)$,

where

$t_{i}(x)=h_{i} \wedge I_{A_{i}}(x), \quad A_{i}=\left\{x_{i}, x_{i+1}, \ldots, x_{n}\right\}$,

$i=1, \ldots, n$.

It can be easily verified that $t_{i} \simeq t_{j} \forall i, j=$ $1, \ldots, n$. By applying the compatibility of $\simeq$ with respect to the maximum and using $\left(a^{\prime}\right)$ and $\left(d^{\prime}\right)$, we can write:

$$
\begin{aligned}
E(h) & =E\left(\bigvee_{i=1}^{n} t_{i}\right)=\bigvee_{i=1}^{n} E\left(t_{i}\right) \\
& =\bigvee_{i=1}^{n} E\left(h_{i} \wedge I_{A_{i}}\right)=\bigvee_{i=1}^{n}\left(h_{i} \wedge E\left(I_{A_{i}}\right)\right) \\
& =\bigvee_{i=1}^{n}\left(h_{i} \wedge g\left(A_{i}\right)\right),
\end{aligned}
$$

and this expression coincides with (1). So, the Sugeno integral with respect to $g$ and $E(\cdot)$ are the same functional:

$E(h)=S_{g}(h) \forall h$.

Uniqueness of $g$ is again evident.

The parallelism between Choquet and Sugeno integrals is complete; both integrals fit the same formal model, and they only differ in the used operators: sum and product for the Choquet integral, maximum and minimum for the Sugeno integral. However, the mathematical properties of these operators give each functional particular features that make them useful in different contexts.

There are other analogies between $E_{g}(\cdot)$ and $S_{g}(\cdot)$. Now we will show how, for the case of possibility measures (defined by Zadeh [26]), the Sugeno integral behaves in a similar way to the Choquet integral, for the case of probability measures.

As we have said above, if the fuzzy measure is a probability $P$, then Choquet integral $E_{P}(\cdot)$ coincides with the mathematical expectation with respect to $P$, and therefore it can be written as:

$E_{P}(h)=\sum_{i=1}^{n} p_{i} h_{i}$

where $p_{i}=P\left(\left\{x_{i}\right\}\right), i=1, \ldots, n$. 
The following proposition gives an analogous expression for $S_{g}(\cdot)$ when $g$ is a possibility measure (see [22] for a proof of that result).

Proposition 3.1. Let $\Pi$ be a possibility measure on $X$ and let $h: X \rightarrow[0,1]$ be a function. The Sugeno integral of $h$ with respect to $\Pi$ can be written as

$S_{\pi}(h)=\bigvee_{i=1}^{n}\left(\pi_{i} \wedge h_{i}\right)$

where $\pi_{i}=\Pi\left(\left\{x_{i}\right\}\right), i=1, \ldots, n$.

Equations (3) and (4) are similar, and they point out the parallelism that exists between probability and possibility with respect to Choquet and Sugeno integrals respectively. To further elucidate this idea, we will prove two propositions that characterize the particular cases of probability and possibility measures.

Proposition 3.2. If in Theorem 3.1 the condition (a) of ordered additivity is replaced by the more restrictive condition of additivity:

$E\left(h+h^{\prime}\right)=E(h)+E\left(h^{\prime}\right), \quad \forall h, h^{\prime}: X \rightarrow \mathbb{R}_{0}^{+}$,

then, and only then, is $E$ the mathematical expectation with respect to a probability measure.

Proof. Necessity: As $g(A)=E\left(I_{A}\right)$, and $E$ is an additive functional,

$$
\begin{aligned}
& \forall A, B \subseteq X \mid A \cap B=\emptyset \\
& \begin{aligned}
g(A \cup B) & =E\left(I_{A \cup B}\right)=E\left(I_{A}+I_{B}\right) \\
& =E\left(I_{A}\right)+E\left(I_{B}\right)=g(A)+g(B),
\end{aligned}
\end{aligned}
$$

and $g$ is a probability measure; therefore $E$ is the mathematical expectation with respect to $g$.

The sufficient condition is evident.

Proposition 3.3. If in Theorem 3.2 the condition $\left(\mathrm{a}^{\prime}\right)$ of ordered F-additivity is replaced by the more restrictive condition of $\mathrm{F}$-additivity:

$E\left(h \vee h^{\prime}\right)=E(h) \vee E\left(h^{\prime}\right), \quad \forall h, h^{\prime}: X \rightarrow[0,1]$,

then, and only then, is $E$ the Sugeno integral with respect to a possibility measure.

Proof. Necessity: Since $g$ is defined as $g(A)=$
$E\left(I_{A}\right)$, and using $I_{A} \vee I_{B}=I_{A \cup B} \forall A, B \subseteq X$, then

$$
\begin{aligned}
g(A \cup B) & =E\left(I_{A \cup B}\right)=E\left(I_{A} \vee I_{B}\right) \\
& =E\left(I_{A}\right) \vee E\left(I_{B}\right)=g(A) \vee g(B) .
\end{aligned}
$$

Therefore $g$ is a possibility measure.

Sufficiency: If $g$ is a possibility measure, by Proposition 3.1 we can write $S_{g}$ as

$$
S_{g}(h)=\bigvee_{i=1}^{n}\left(h_{i} \wedge g\left(\left\{x_{i}\right\}\right)\right)
$$

Then,

$$
\begin{aligned}
S_{g}\left(h \vee h^{\prime}\right) & =\bigvee_{i=1}^{n}\left(\left(h_{i} \vee h_{i}^{\prime}\right) \wedge g\left(\left\{x_{i}\right\}\right)\right) \\
& =\bigvee_{i=1}^{n}\left(h_{i} \wedge g\left(\left\{x_{i}\right\}\right)\right) \vee \bigvee_{i=1}^{n}\left(h_{i}^{\prime} \wedge g\left(\left\{x_{i}\right\}\right)\right) \\
& =S_{g}(h) \vee S_{g}\left(h^{\prime}\right) .
\end{aligned}
$$

These results support the important conclusion that the mathematical expectation for probability measures is extended to fuzzy measures as a consequence of weakening the additivity property, thus obtaining an ordered additive functional, i.e. the Choquet integral. It is also evident from these results that the Sugeno integral for general fuzzy measures is a weakening of the Sugeno integral for possibility measures, replacing the F-additivity property by the weaker ordered F-additivity (for more details, see [6]).

One of the differences between the two integrals that may be noted is that the Sugeno integral does not make any sense for functions values outside the interval $[0,1]$, whereas the Choquet integral makes sense for any nonnegative function (and also for any real function, but that is not important here; see [16] for details). Several attempts to modify the former's definition to extend this nonlinear functional to $\mathbb{R}_{0}^{+}$in a linear way (see [12]) have been strongly criticized (see [13]).

Two questions remain to be considered.

One involves the characterization theorems of the two integrals considered in an attempt to further refine them. Is it possible to remove some of the conditions, without compromising the present characterizations?

Since both integrals fit the same formal model, it would also be worthwhile to investigate the 
possibility of defining other fuzzy integrals following the same model but using other operators, and to analyze what properties such new functionals would verify.

Both questions will be object of further works.

Finally, another important open problem that should be considered in the future is the extension to the case of infinite universes of the results obtained in this paper for the finite case. Some results concerning the convergence properties of monotone expectation (see [5]) could be useful for this task.

\section{References}

[1] N. Batle and E. Trillas, Entropy and fuzzy integral, $J$. Math. Anal. Appl. 69 (1979) 469-474.

[2] $M$. Berres, On a multiplication and a theory of integration for belief and plausibility functions, $J$. Math . Anal. Appl. 121 (1987) 487-505.

[3] M.J. Bolaños, Caracterización de medidas y valoraciones difusas a partir de medidas ordinarias, Thesis, Universidad de Granada (1984).

[4] M.J. Bolaños, M.T. Lamata and S. Moral, La esperanza monótona: una generalización de la esperanza probabilística, $X V$ Reunión Nacional de Estadística e Investigación Operativa, Asturias (Spain) (1985) 57-64.

[5] M.J. Bolaños, L.M. de Campos and A. González, Convergence properties of the monotone expectation and its application to the extension of fuzzy measures, Fuzzy Sets and Systems 33 (1989) 201-212.

[6] L.M. de Campos, Caracterización de medidas e integrales difusas a partir de probabilidades, Thesis, Universidad de Granada (1987).

[7] L.M. de Campos and M.J. Bolaños, Representation of fuzzy measures through probabilities, Fuzzy Sets and Systems 31 (1989) 23-36.

[8] G. Choquet, Theory of capacities, Ann. Inst. Fourier 5 (1953) 131-295.
[9] A.P. Dempster, Upper and lower probabilities induced by a multivalued mapping, Ann. Math. Statist. 38 (1967) 325-339.

[10] P.J. Huber, The use of Choquet capacities in statistics, Bull. Internat. Statist. Inst. 45 (1973) 181-188.

[11] P.J. Huber, Robust Statistics (Wiley, New York, 1981).

[12] A. Kandel, On fuzzy statistics, in: M.M. Gupta, R.K. Ragade and R.R. Yager, Eds., Advances in Fuzzy Sets Theory and Applications (North-Holland, Amsterdam, 1979) 181-198.

[13] E.P. Klement and D. Ralescu, Nonlinearity of the fuzzy integral, Fuzzy Sets and Systems 11 (1983) 309-316.

[14] G.J. Klir, Architecture of Systems Problem Solving (Plenum Press, New York, 1985).

[15] R. Kruse, Fuzzy integrals and conditional fuzzy measures, Fuzzy Sets and Systems 10 (1983) 309-313.

[16] M.T. Lamata, Modelos de decisión con información general, Thesis, Universidad de Granda (1985).

[17] T. Murofushi and M. Sugeno, An interpretation of fuzzy measures and the Choquet integral as an integral with respect to a fuzzy measure, Fuzzy Sets and Systems 29 (1989) 201-227.

[18] D. Ralescu and G. Adams, The fuzzy integral, J. Math. Anal. Appl. 86 (1980) 176-193.

[19] $\mathrm{Ph}$. Smets, The degree of belief in a fuzzy event, Inform. Sci. 25 (1981) 1-19.

[20] F. Suárez and P. Gil, Two families of fuzzy integrals, Fuzzy Sets and Systems 18 (1986) 67-81.

[21] M. Sugeno, Theory of fuzzy integrals and its applications, Thesis, Tokio Inst. of Technology (1974).

[22] M.A. Vila and M. Delgado, On medical diagnosis using possibility measures, Fuzzy Sets and Systems 10 (1983) 211-222.

[23] P. Wakker, Additive Representations of Preferences (Kluwer, Dordrecht-Boston, 1989).

[24] S. Weber, $\perp$-Decomposable measures and integrals for archimedean t-conorms $\perp$ J. Math. Anal. Appl. 101 (1984) 114-138.

[25] S. Weber, Two integrals and some modified versions Critical remarks, Fuzzy Sets and Systems 20 (1986) 97-105.

[26] L.A. Zadeh, Fuzzy sets as a basis for a theory of possibility, Fuzzy Sets and Systems 1 (1978) 3-28.

[27] Wang Zi-Xiao, Fuzzy measures and measures of fuzziness, J. Math. Anal. Appl. 104 (1984) 589-601. 